\title{
A NOTE ON SPACES WITH NORMAL PRODUCT WITH SOME COMPACT SPACE
}

\author{
R. W. THOMASON ${ }^{1}$
}

ABSTRACT. For compact $X$ with $\log |X| \geqq \aleph, X \times Z$ is normal only if $Z$ is $\boldsymbol{x}$-collectionwise normal. If $Z$ is also semimetric or $\aleph$-metacompact, it is then $\aleph$-paracompact.

All spaces herein are to be Hausdorff. The density function is denoted $d(\quad)$.

LEMMA 1. If $Y$ is a nondegenerate $A E$ for $X$, and $Y^{X}$ has the compactopen topology, $d\left(Y^{X}\right) \geqq d(Y) \cdot \log |X|$.

Proof. $\prod Y_{x}, x \in X$, has density $d(Y) \cdot \log |X|[3$, Theorem 4.5]. $Y \boldsymbol{X}$ with the $p$-topology is a dense subspace of $\prod Y_{x}$, so its density is no less. The result follows as the compact-open topology is finer than the $p$-topology.

Henceforth, $Y^{X}$ will always have the compact-open topology. From Lemma 1, we see that if $Y$ is a metric nondegenerate AE for normal spaces, and $X$ is a compact space with $|X|>2^{N_{0}}, Y^{X}$ is not even an ANE for perfectly normal spaces. For $d\left(Y^{X}\right)>\aleph_{0}$, but $Y^{X}$ is metric, and E. Michael has shown that metric ANE's for Bing's perfectly normal, but not uncountably collectionwise normal, spaces must be separable [6, Theorem 3.1]. (Incidentally, this shows the "only if" parts of Problems 5.7 and 5.8 of Chapter XV of Dugundji's text [2] are incorrect.) We generalize this as follows:

LEMMA 2. If $Y$ is a metric ANE for $Z, Z$ is $d(Y)$-collectionwise normal.

Proof (CF. Proposition 5.1 of [6]). Since $Y$ is metric, its cellularity is attained and equals its density [1, Theorem 4]. Given any discrete collection $\left\{K_{\alpha}\right\}$ of no more than $d(Y)$ closed subsets of $Z$, we may then take as many disjoint nonempty open subsets $\left\{U_{\alpha}\right\}$ of $Y$. Let $f$ be the continuous extension over an open neighborhood of $\bigcup K_{\alpha}$ of the map from

Received by the editors October 6, 1972 and, in revised form, January 11, 1973.

AMS (MOS) subject classifications (1970). Primary 54A25, 54D15, 54D20.

Key words and phrases. Compact, normal, AE, $\boldsymbol{\aleph}$-collectionwise normal, $\boldsymbol{x}$ paracompact.

${ }^{1}$ The author is indebted to Professor D. A. Moran of Michigan State University for his kind assistance.

(c) American Mathematical Society 1974 
$\cup K_{\alpha}$ to $Y$ sending each $K_{\alpha}$ to a point in the corresponding $U_{\alpha}$. Then $\left\{f^{-1}\left(U_{\alpha}\right)\right\}$ is a collection of disjoint open sets in $Z$ separating $\left\{K_{\alpha}\right\}$.

TheOREM. Let $X$ be compact, $\log |X| \geqq \aleph$. Then if $Z \times X$ is normal, $Z$ is $\boldsymbol{\aleph}$-collectionwise normal. If $Z$ is also $\mathbf{\aleph}$-metacompact or semimetric, it is then $\boldsymbol{\aleph}$-paracompact.

Proof. Let $A$ be a closed subset of $Z$. If $f: A \rightarrow R^{X}$ is continuous, so is its associate $f^{*}: A \times X \rightarrow \boldsymbol{R}$. We may extend $f^{*}$ to a continuous map $F^{*}: Z \times X \rightarrow R$, and its associate $F: Z \rightarrow R^{X}$ is a continuous extension of $f$. Thus $\boldsymbol{R}^{X}$ is an AE for $Z$; by Lemma $1, d\left(\boldsymbol{R}^{X}\right) \geqq \mathcal{N}$; and now by Lemma 2 , $Z$ is $N$-collectionwise normal.

Bearing in mind that point-finite refinements may be made precise [2, Theorem VIII 1.4], one may adapt Michael's proof of Theorem 2 in [5] to show spaces both $\boldsymbol{\aleph}$-metacompact and $\boldsymbol{N}$-collectionwise normal are $\boldsymbol{N}$-paracompact. Similarly, adapt McAuley's proof of Lemma 2 in [4] to handle the case where $Z$ is semimetric.

Note if $\log |X| \geqq 2^{d(Z)}$ in the above, we get $Z$ is collectionwise normal, for $Z$ has no discrete collection of more than $2^{d(Z)}$ subsets [2, VII 3, Example 3].

Morita has shown $Z$ is $\boldsymbol{N}$-paracompact and normal iff $Z \times I^{\aleph}$ is normal [7, Theorem 2.4]. The above theorem generalizes the "iff" part of this, replacing $I^{\aleph}$ with any compact space of equal cardinality, but draws a weaker conclusion.

\section{REFERENCES}

1. W. W. Comfort, A survey of cardinal invariants, General Topology and Appl. 1 (1971), 163-199. MR 44 \#7510.

2. J. Dugundji, Topology, Allyn and Bacon, Boston, Mass., 1966. MR 33 \#1824.

3. I. Juhasz, Cardinal functions in topology, Math. Centrum, Amsterdam, 1971.

4. L. F. McAuley, A note on complete collectionwise normality and paracompactness, Proc. Amer. Math. Soc. 9 (1958) 796-799. MR 20 \#6086.

5. E. Michael, Point-finite and locally finite coverings, Canad. J. Math. 7 (1953), 275-279. MR 16, 1138.

6. - Some extension theorems for continuous functions, Pacific J. Math. 3 (1953), 789-806. MR 15, 547.

7. K. Morita, Paracompactness and product spaces, Fund. Math. 50 (1961/62), 223-236. MR 24 \#A2365.

Department of Mathematics, Michigan State University, East Lansing, Michigan 48823

Current address: Department of Mathematics, Princeton University, Princeton, New Jersey 08540 